\title{
Ameliorating chronic pain: opioids, adjunct and alternative therapies
}

\author{
M. C. Powanda ${ }^{1}$ P. J. Berry ${ }^{1}$ - K. D. Rainsford ${ }^{1}$
}

Received: 17 July 2016/Accepted: 16 October 2016/Published online: 24 October 2016

(C) Springer International Publishing 2016

"The aim of the wise is not to secure pleasure, but to avoid pain". Aristotle seems to sum up the problem of achieving relief from pain, especially chronic pain, while avoiding addiction. Allowing a person or animal to suffer debilitating pain when relief is possible seems so repugnant to society that it is considered acceptable that the terminally ill may be provided with massive doses of drugs to ease their living and dying. However, for those who are not terminally ill there is concern that patients seeking relief from severe chronic pain may become addicts. This concern increases the reluctance to prescribe opioids, which can lead to the under treatment of pain. It is a formidable health problem that is causing many people to now ask that if those at the foreseeable end of their lives can be spared unnecessary pain, what about the patients who are still active and want to continue to be productive?

The successful treatment of inflammatory diseases and the effective amelioration of pain, whatever is its source, are critical to achieving a quality of life that fosters productivity and facilitates social interaction. Inflammopharmacology publishes papers on all aspects of inflammation and associated pain, as well as its pharmacological control. These include articles on new and existing analgesic agents, along with mechanisms of neuroinflammation and pain. While it seems reasonable to provide patients in severe chronic pain appropriate relief, including opioids, it is clear that because of growing concerns about potential addiction, limited access to such drugs may occur even when their use is warranted. Thus, the Journal plans to create a forum which will focus on the appropriate clinical use of opioids and possible approaches to increase their safer use by patients, such as access to naloxone or buprenorphine, along with agents to treat constipation. In addition, the Journal will concentrate on whether or not there are data to support patients taking, and family doctors recommending/prescribing, viable alternatives to opioids. Thus, it is critical to determine what evidence exists that there are indeed, viable alternatives that will at least ameliorate pain.

While previously the journal has published nonclinical studies of drugs and natural products to treat pain and inflammation, the emphasis of this forum will be articles of clinical trials of conventional drugs, new chemical or biological entities, natural products, forms of exercise, e.g., yoga, tai chi and modulation of mindset (hypnotherapy, meditation, antidepressants) alone or in combination, that appear to be clinically useful in reducing or eliminating pain and promoting function. Reviews, as well as previously unpublished clinical data, including negative clinical data, will be considered. Supporting nonclinical data suggesting the rationale (efficacy and safety) for conducting the clinical trial(s) will be considered for publication, but only when submitted with the clinical trial data.

\footnotetext{
M. C. Powanda

powanda@mpbiomed.com

1 M/P Biomedical Consultants LLC, Mill Valley, CA, USA
} 